\title{
Physiological Effects of Spaceflight/Unloading and the Mitigating Effects of Flywheel-Based Resistive Exercise
}

\author{
Prashant Parmar ${ }^{1}$, Rachel Perry ${ }^{2}$, Greta Cesarz ${ }^{3}$, Alex Roberts ${ }^{3}$, Houston Hardman ${ }^{2}$, and John F. Caruso ${ }^{3}$ \\ ${ }^{1}$ School of Community Medicine, University of Oklahoma, Tulsa Oklahoma 74104; ${ }^{2}$ Exercise and Sport Sciences \\ Program, The University of Tulsa, Tulsa Oklahoma 74104; ${ }^{3}$ Dept. Health and Sport Sciences, The University of \\ Louisville, Lousiville Kentucky 40292
}

\begin{abstract}
The deleterious effects of spaceflight encompass numerous physiological effects that undermine long-term goals of manned round-trip missions to Mars. Among the greater losses are to the human musculoskeletal system due to limited mechanical/load-bearing activity. In-flight exercise and nutritional countermeasures seek to reduce physiological losses. Restoration of mechanical/load-bearing activity in microgravity is achieved with flywheel-based exercise hardware. Research with spaceflight analogs showed exercise done with flywheel-based devices abated muscle mass and strength losses with modest increases in net energy costs. This led to the installment of flywheel-based hardware on The International Space Station (ISS). To date, exercise with flywheel-based hardware has reduced musculoskeletal losses, with more
\end{abstract}

Key words: Countermeasures, Nutrition and Metabolism, Body Mass, Cardiovascular Changes, Oxidative Stress, Muscle Atrophy, Strength Loss, Bone Demineralization, Mechanical Loading, Strain Rates

Correspondence to: John F. Caruso Department of Health and Sport Sciences The University of Louisville Louisville Kentucky 40292

Telephone: 502-852-6648

E-mail: john.caruso@louisville.edu success achieved for muscle-, versus bone-based, outcomes. In-flight exercise may better address bone losses with hardware that imparts high rates of impulse loading to the engaged musculoskeleton.

\section{INTRODUCTION}

\section{Deleterious Effects Of Spaceflight/Unloading}

Microgravity $(\mu g)$ poses unique challenges to a species that evolved entirely within a loadbearing eugravitational environ-ment. Numerous changes occur as humans are exposed to $\mu g$ that have far-ranging effects on their health (Gopalakrishnan et al., 2010). Some even question if long-term manned round trip missions to Mars are even tenable (Hawkey, 2005; Riley, 1999). Adverse changes to health and human physiology were seen post-flight from even the earliest shortduration American and Soviet spaceflights. A stimulus for some adverse changes is the disruption of sufficient mechanical and loadbearing activity to the lower body weight-bearing musculoskeleton. Such changes, and the adverse events anticipated from longer missions, led to the deployment of in-flight countermeasures to combat the detrimental effects of $\mu g$. This review summarizes nutritional and physiological changes seen with $\mu g$, and then addresses the degree of mitigation attained from spaceflight and $\mu g$ simulation models with flywheel-based hardware. The use of flywheels to impart mechanical loads 
helped limit musculoskeletal losses in groundbased $\mu g$ simulation, which in turn led their use on in-flight hardware now on the ISS.

\section{Nutritional and Metabolic Changes Associated with $\mu g$}

Since the Skylab missions, space agencies are aware of nutritional concerns seen with $\mu g$ (Smith and Zwart, 2008). Spaceflight causes fluid shifts that lead to motion sickness, taste and smell pertubations, and an altered hormonal status (Hargens and Richardson, 2009; Matsumoto et al., 2011; Moore et al., 2010; Smith et al., 2013). For instance, $\mu g$ causes a cephalic body fluid shift at the expense of plasma volume (Hargens and Richardson, 2009; Matsumoto et al., 2011; Moore et al., 2010; Smith et al., 2013). The increase in fluids may change the perception for the aforementioned cephalic sensory stimuli, while the lower plasma volume caused by fluid shifts can elicit higher blood concentrations for some hormones. Spaceflight analogs induce physiological losses that typically increase with the longer periods of unloading (Baldwin et al., 1996; Caruso et al., 2005b; Zwart et al., 2012). For instance, bed rest evoked progressively greater weight losses over 30, 60, and 90 days; while unilateral limb suspension evoked progressively greater strength losses after 20 and 40 days of unloading (Caruso et al., 2005b; Zwart et al., 2012). $\mu g$ typically elicits body weight losses (Matsumoto et al., 2011). Significant body weight decreases occurred in astronauts, with some even exceeding $5 \%$ of pre-flight body mass. A similar degree of individual losses also occurred after a 42-day bed rest (Blanc et al., 1998). Body mass losses are exacerbated by other factors, such as losses in appetite (Stein, 2000). Adequate nutrition may help address some of the adverse effects seen with $\mu g$ and abate the energy deficits caused by in-flight exercise (Matsuo et al., 2012; Parmar et al., 2015; Smith and Zwart, 2008). Actual energy consumption on early spaceflights was low, while ISS missions had crewmembers routinely eating at $90 \%$ or above their recommended intakes, which maintained body mass (Smith et al., 2013). Yet higher energy intakes impose added concerns for long-duration missions: when each kilogram of mass must be accounted for during takeoff and storage, how much of this valuable space must be dedicated to foodstuffs to keep astronauts healthy? Once a balance between food intake, energy costs, and the impact exercise has on weight loss are found, only then can optimum in-flight countermeasures to weight loss be devised (Matsuo et al., 2012).

Relationships between exercise in $\mu g$ and body mass losses are paradoxical, because while exercise abates muscle mass and strength deficits, the resultant energy costs hasten weight loss (Matsomoto et al., 2011; Stein, 2000; Wickman et al., 2011). Unfortunately, when in-flight exercise is deployed, the resultant energy costs are not always accounted for. Human metabolic rates rise sharply in $\mu g$ (Matsuo et al., 2012; Stein, 2000; Wickman et al., 2011). In-flight peak and average energy costs for extravehicular activities of 500 and 200-250 kcal/hour, respectively, were reported (Stein, 2000; Whittle, 1979; Wickman et al., 2011). A 71-kilogram astronaut has an estimated in-flight energy expenditure of 2938 kcals/day, which entails an allowance for one hour of exercise at a metabolic cost of 490 kcals (Wickman et al., 2011). With higher metabolic rates, in-flight accrual and removal of body heat is another health concern, as post-flight hyperthermia is common (Convertino and Tsiolkovsky, 1990). Sweat rates decline with longer flights, which increases the thermal stress to crewmembers (Convertino and Tsiolkovsky, 1990). For instance, 14 days of bed rest, with exogenous acetylcholine administration before and after the intervention, saw a significant average decline in sweat rate of $33 \%$ versus prebed rest values (Crandall et al., 2003). The degree of dehydration seen with 14 days of $\mu g$ approximates a $10 \%$ loss in plasma volume (Ogawa et al., 2009). Submaximal cycle ergometery tests done after 115 days of $\mu g$ produced a significant average decline in sweat rate of $75 \%$ versus pre-flight values (Fortney et al., 1998). Sweating in $\mu g$ forms a film over the skin that impedes convective heat loss, which can impair an astronaut's ability to perform mission objectives (Convertino, 1990; Fortney et al., 1998). The higher thermal stress from elevated metabolic rates is compounded when exercise is done in $\mu g$.

Successful in-flight exercise requires they impose little increase to metabolic rates above those that naturally occur in $\mu \mathrm{g}$. This is made difficult with limited choices in exercise 
hardware, as well as finite amounts of $\mathrm{O}_{2}$ and food; in regards to the latter, life support requirements per crewmember and day provide $0.7 \mathrm{~kg}$ of dry food in $\mu \mathrm{g}$ (Matsumoto et al., 2011; Matsuo et al., 2012; Wickman et al., 2011). For longer missions, exercise with modest energy costs will reduce demands on ISS food supplies and the costs to replenish such materials (Matsomoto et al., 2007; Matsuo et al., 2012). Presumably the elevated metabolic rates and hyperthermia seen in humans exposed to $\mu g$ cause higher energy costs for in-flight exercise than when the same types of activity occur on Earth (Matsumoto et al., 2011; Matsuo et al., 2012; Stein, 2000; Stein et al., 1999; Wickman et al., 2011). In-flight aerobic exercise evokes negative energy balances (Dudley et al., 1991) and removal of large amounts of its byproducts $\left(\mathrm{CO}_{2}\right.$, heat $)$ poses added concerns. The link between exercise and energy deficits is exacerbated by reduced protein synthesis (Stein, 2000) and the absence of mechanical-loading (Shenkman and Nemirovskaya, 2008) to evoke vast muscle atrophy and strength losses (Fitts et al., 2000; Wickman et al., 2011).

Insufficient energy intakes in $\mu \mathrm{g}$ may deprive crewmembers of essential nutrients, such as minerals and fat-soluble vitamins (Smith and Zwart, 2008; Smith et al., 2013). There is much agreement on the consequences of vitamin deficiencies in an environment where humans are not protected from radiation by the Earth's magnetic fields (Smith and Zwart, 2008; Smith et al., 2013). Vitamins A, D, E, and $K$ are critical to human health. Vitamin D, in particular, had the most pronounced deficiencies from $\mu g$, causing NASA to add vitamin D supplements to dietary plans (Smith et al., 2013). Since vitamin D impacts calcium absorption and bone health, it now serves as a nutritional countermeasure. Minerals perhaps most impacted by $\mu g$ include calcium, phosphorous, and magnesium (Morgan et al., 2012; Smith et al., 2012c). For instance, calcium accrual within unloaded muscle activates calpains to initiate the myofibrillar protein catabolism that contribute to muscle atrophy and strength loss (Shenkman and Nemirovskaya, 2008). Iron also changes, with heme iron leaving microcytic red blood cells and reduced bloodstream levels of transferrin (usually an acute phase reactant), which can lead to some symptoms of mild anemia as iron stores redistribute themselves (Smith and Zwart, 2008). Finally, of critical importance are the vitamin deficiencies seen with megaloblastic anemias, namely folate and B12, which can cause long-term retinal damage and are a critical health issue in $\mu g$ (Zwart et al., 2012).

\section{Physiological Changes Associated with $\mu g$}

The adverse changes humans experience in $\mu g$ are interrelated to the higher metabolic rates seen with spaceflight. They include hormonal and cardiovascular changes, oxidative damage, musculoskeletal losses. Concerted efforts were undertaken to devise in-flight treatments to address each of these areas. They including limiting energy costs for the American space program's aerobic exercise regimen (Moore et al., 2010), to artificial $g$ induction (Iwate, 2005), to customized devices with the goal of acting as an all-in-one countermeasure (Streeper et al., 2011).

\section{Hormonal changes associated with $\mu \mathrm{g}$}

$\mu \mathrm{g}$ induces numerous hormonal changes reminiscent of a chronically stressed state. Elevated cortisol, and decreased testosterone, each result from space flight/unloading (Crucian et al., 2000; Ronca et al., 2014; Smith et al. 2012b; Stowe et al., 2001; Stowe et al., 2003; Strollo 1999). For instance as compared to their pre-flight values, in-flight testosterone levels for four male astronauts flown on the ten-day 1993 Spacelab D2 mission were significantly less (Strollo, 1999). Significantly suppressed testosterone levels were also seen in male astronauts flown on subsequent short-term (12-13 day) missions, as well as for longer (48-215 days) stays on the ISS, as measured 0-1 days post-flight (Smith et al., 2012b). Particularly for shorter periods of $\mu \mathrm{g} / \mathrm{unloading}$, temporal testosterone changes are thought to assist in the body's adaptation to such novel environments (Strollo, 1999; Smith et al. 2012b). In contrast to testosterone, cortisol levels increase in actual and simulated $\mu g$ (Ronca et al., 2014). A nine-day spaceflight yielded significant cortisol increases in seven astronauts, as compared to their pre-flight values (Stowe et al., 2001). Urinary cortisol was significantly elevated in 17 astronauts after 10-18 days of $\mu \mathrm{g}$, as compared to their pre-flight values (Crucian et al., 2000). Furthermore in 28 crewmembers exposed 
to 16 days of $\mu \mathrm{g}$, plasma cortisol was significantly higher than their pre-flight values (Stowe et al., 2003). It was concluded longer flights may induce changes to glucocorticoids upon landing commensurate to the duration and stress incurred from individual missions (Stowe et al., 2003). Chronically high plasma cortisol levels increase peripheral insulin resistance and elevate triglycerides that may lead to cardiac and oxidative damage (Hargens and Richardson, 2009).

\section{Cardiovascular changes associated with $\mu \mathrm{g}$}

Cardiovascular system responses to $\mu g$ include acute and chronic changes thought necessary for humans to exist in such a novel environment. While the cardiovascular system adapts rather quickly to $\mu \mathrm{g}$, it is less able to effectively compensate for sudden re-exposure to $g$ upon return to Earth (Ferretti and Capelli, 2009). Orthostatic intolerance from reduced stroke volume upon returning to Earth is one of the more common cardiovascular concerns, as some astronauts experience syncope from limited blood to the brain, while other also note impaired venous return from their lower extremities that undermines cardiac output (Hargens and Richardson, 2009). In-flight cardiovascular changes from longer flights include venous hypotrophy produced from vascular wall remodeling (Ferretti and Capelli, 2009). On Earth, circulation to the extremities is assisted by $g$ and substantial arterial smooth muscle involvement (Delp, 2007; Hargens and Richardson, 2009). Yet during spaceflight the body instantly compensates to a reduced plasma volume through alterations in serum proteins (Smith and Zwart, 2008). Unfortuantely a fluid loading countermeasure alone does not restore blood volume (Smith and Zwart, 2008). The result of these adaptations to $\mu \mathrm{g}$ is cardiovascular function, $\mathrm{O}_{2}$ uptake and autonomic function remain may relatively similar to pre-flight values. However upon return to Earth the combination of the sudden rush of fluids into lower extremity veins with their more compliant walls, a reduced stroke volume and lower baroreflexes all contribute to hypotension and orthostatic intolerance (Ferretti and Capelli, 2009; Smith and Zwart 2008).

The numerous cardiovascular changes humans experience in $\mu g$ are actually designed to preserve $\mathrm{O}_{2}$ uptake as much as possible, with the result it is possible to see no decrease in aerobic capacity if a sufficient amount of concurrent inflight exercise is performed (Ferretti and Capelli, 2009). Yet upon return to Earth, $\mathrm{VO}_{2}$ max drops significantly for two reasons: cardiovascular deconditioning and myocardial atrophy. When humans return to Earth their hearts cannot adapt rapidly to $g$, and decreased muscle mass and myofiber conversion to glycolytic fibers lowers their cardiac efficiency. In regards to countermeasures to cardiovascular deconditioning, it is essential to note cardiac decompensation cannot be restored by fluid loading alone. Another potential in-flight countermeasure is intermittent artificial $g$, which may allow a more gradual adaptation to Earth, as opposed to the sudden resumption of weightbearing activity astronauts now experience (Yang et al., 2011; Hargens and Richardson, 2009). Some imply periodic in-flight episodes of artificial gravity induction will limit cardiovascular and $\mathrm{VO}_{2}$ max changes (Ferretti and Capelli, 2009). Finally a ground-based study suggests high-intensity cycle ergometry may better attenuate cardiovascular and metabolic changes incurred in $\mu g$ (Matsuo et al., 2012).

\section{Oxidative damage associated with $\mu \mathrm{g}$}

There are also concerns of elevated oxidative damage from hypercortisolemia that tends to rise with the rigor and duration of missions (Crucian et al., 2000; Ronca et al., 2014; Stowe et al., 2001; Stowe et al., 2003), and heightened free radical formation seen with longer (Smith et al., 2001; Smith et al., 2005), but not shorter (17-day) (Stein and Leskiw, 2000), spaceflights. Furthermore, similar to $\mathrm{VO}_{2}$ max changes, the return to Earth increase the likelihood of increased oxidative damage, as multiple systems compete to repair after exposure to $\mu g$ (Stein, 2002). New research aimed at limiting the oxidative damage associated with $\mu g$ are warranted.

\section{Musculoskeletal changes associated with $\mu \mathrm{g}$}

Among the greater losses from $\mu \mathrm{g} / \mathrm{unloading}$ are to the musculoskeletal system (Fitts et al., 2000; Riley, 1999); such decrements impair in-flight procedural tasks, mission goals and post-flight astronaut health upon their return to Earth. Similar 
changes occur to ground-based $\mu g$ analogs. Muscle decrements primarily occur to the lower limbs, with volume losses of up to $16 \%$ to the calf and $7 \%$ to the thigh from a six-month stay aboard the ISS (Gopalakrishnan et al., 2010). The stimulus for such losses is the disruption of sufficient mechanical and load-bearing activity. Yet other factors contribute to these losses. For instance the hypercortisolemic state seen with $\mu \mathrm{g} /$ unloading promotes muscle breakdown, with the greatest proteolysis seen at the initial start of spaceflight or bed rest, and thereafter catabolism proceeds at slower rates (Smith and Zwart, 2008). A 28-day bed rest study, whereby exogenous cortisol was administered to better simulate $\mu \mathrm{g}$, increased sarcolemmal triglycerides and peripheral insulin resistance, which each contribute to the functional losses for unloaded muscle (Cree et al., 2010). Yet cortisol changes for this unloading model may be considerably different than what astronauts encounter in $\mu g$, particularly if they also engage in exercise. $\mu g$ and exercise individually increase endogenous cortisol secretion; combining the two, as with in-flight exercise, may evoke different changes than bed rest (Caruso et al., 2010; Cree et al., 2010; Ronca et al., 2014).

Spaceflight/unloading elicits numerous changes, which include shifts to more glycolytic myofibers (Trappe et al., 2009). Such changes lead to greater fatigue that jeopardize in-flight tasks and the ability to perform emergency egress. The magnitude of strength losses from spaceflight/unloading exceeds those for muscle atrophy. The lower body weight-bearing muscles incur the greatest losses (Belavy` et al., 2009; Fitts et al., 2000; Riley et al., 2002; Rittweger et al., 2006). Spaceflights of 5-11 days saw knee extensor force decline $15 \%$ versus pre-flight values (Edgerton et al., 1995). Vastus lateralis biopsies 5-11 days post-flight showed crosssectional area losses to slow (-15\%) and fast muscle $(-22 \%)$ fibers as well as higher expression of fast myosin heavy chains (Edgerton et al., 1995). Seven-day bed rests evoked significant (3\%) thigh muscle volume and knee extensor strength losses (Ferrando et al., 1995; Friman and Hamrin, 1976; Hayes et al., 1992). Unilateral limb suspension for 20 days led to a $7 \%$ loss to unloaded knee extensor cross-sectional area (Schultze et al., 2002). A 30-day bed rest evoked higher (-8\%) thigh cross-sectional area losses; muscle biopsies showed significant losses by fiber (slow twitch -11\%, fast twitch -18\%) type (Hikida et al., 1989). Unilateral limb suspension for 28 days evoked unloaded thigh cross-sectional area losses $(\sim-7 \%)$ similar to a 30-day bed rest (Berg et al., 2007; Tesch et al., 2004). Longer unilateral limb suspension studies increased knee extensor ( -9-16\%) atrophy (Alkner and Tesch, 2004a; Alkner and Tesch, 2004b; Berg et al., 2007; Caruso et al., 2004b; 1992; Le Blanc et al., 1992). Unloading-induced strength losses exceed those for atrophy due to the role neural factors play in force output (Fitts et al., 2000; Riley et al., 2002). A 14-day bed rest led to a $9 \%$ knee extensor torque loss (Bamman et al., 1998). The level of knee extensor losses (-20\%) from a 30-day bed rest was like that from a 28-day space flight (Convertino et al., 1989). Unilateral limb suspension for 20-25 days evoked unloaded knee extensor torque deficits of $-17 \%-21 \%$ less than pre-unloading values (Berg et al., 1991; Berg et al., 2007; Schultze et al., 2002). Forty days of unilateral limb suspension (-17-22\%) and a 90day bed rest (-31-60\%) led to greater knee extensor force deficits (Alkner and Tesch, 2004b; Caruso et al., 2004b).

Unlike muscle, bone mass and density decrements occur at slower rates; yet such losses are more difficult to recover from and may persist long after astroanuts return to Earth (Carpenter et al., 2010). Thus bone losses represent a vital risk factor to be mitigated. Both $\mu g$ and bed rest led bone resorption rates to rise by up to $150 \%$, while bone formation was unchanged, to produce a net resorption of bone and the loss calcium stores (Morgan et al., 2012; Smith et al., 2012b). Of note this calcium loss was only partially compensated through nutrition interventions due to the lack of upregulation of bone formation (Morgan et al., 2012; Smith et al., 2012b). Bone mineral density losses from $\mu \mathrm{g}$ /unloading occur at rates of $1-5 \%$ per month (Holick, 1998; Lang et al., 2004; LeBlanc et al., 1992; Sibonga et al., 2007). Trabecular bone is most prone to in-flight losses (Collet et al., 1997; Lang et al., 2004). A 21-day unloading period evoked small (-0.9\%) yet significant bone mineral content losses to the unloaded tibia (Rittweger et al., 2006). A 30-day bed rest led to significant losses $(-3.7 \%)$ in summed tibia-fibula cross-sectional area 
(Convertino et al., 1989). A 56-day bed rest caused significant bone mineral content losses to the distal tibia (Rittweger et al., 2006). Changes to vertebral bone mineral density showed significant deficits after five (-3.2\%) and seven (-6.6\%) months of spaceflight (Oganov et al., 1990). A 17week bed rest evoked calcaneal $(-10.4 \%)$ bone mineral density losses (LeBlanc et al., 1992). Other significant losses from the 17-week study included the lumbar spine (-3.9\%), femoral neck and trochanter (-3.6\% and $-4.6 \%$ respectively), as well as the tibia (-2.2\%) (LeBlanc et al., 1992). It was thought hip and spine losses may reach $10 \%$ annually without countermeasures (LeBlanc et al., 1992). Since individual losses could exceed this value, countermeasures to musculoskeletal losses were recommended for long-term flights (LeBlanc et al., 1992).

\section{MITIGATING PHYSIOLOGICAL LOSSES WITH FLYWHEEL-BASED HARDWARE}

In-flight countermeasures to reduce the adverse physiological changes seen with $\mu g$ are essential if long-term space travel is to include round-trip manned missions to Mars. The stimulus for some losses is the lack of sufficient mechanical and load-bearing activity to the lower body weight-bearing musculoskeleton. (Dudley et al., 1991; Fitts et al., 2000). To date, in-flight aerobic exercise has not abated musculoskeletal losses, and mitigated only some cardiovascular changes incurred from $\mu g$ (Dudley et al., 1991; Matsuo et al. 2012). Furthermore aerobic exercise elicits high energy expenditures, which only exacerbates the body mass losses seen with spaceflight/unloading (Matsuo et al. 2012). It appears in-flight exercise must impart sufficient mechanical and load-bearing activity at minimal metabolic cost in an environment where traditional $g$-based exercise hardware is inoperable (Parmar et al., 2015). Fortunately exercise with flywheel-based hardware ameliorated lower body muscle mass and strength losses in unloading models. Such improvements occurred with (Caruso et al. 2004b; Caruso et al. 2005b) and without (Tesch et al., 2004) concurrent administration of pharmaceutical countermeasures.

Research on in-flight exercise noted stationary cycling against mechanical loads of 80100 watt $\cdot \mathrm{min}^{-1} \cdot \mathrm{kg}$ lean body $\mathrm{mass}^{-1} \cdot$ day $^{-1}$ preserved knee and ankle extensor muscle mass (Whittle, 1979). Yet when compared to unilateral leg press data obtained from flywheel-based hardware (Caruso et al., 2004a), it appears bilateral activity for that exercise can achieve similar mechanical loading values at a far smaller training volume and net energy cost. Groundbased research showed flywheel-based hardware imposes high levels of resistance that users overcome as repetitions proceed at slow rates of movement, yet its net energy costs are rather modest and far less than for aerobic exercise (Caruso et al., 2003; Caruso and Hernandez, 2002; Dudley et al., 1991; Moore et al., 2010). A 3-set 8-repetition leg press protocol on flywheel-based hardware yielded a mean net energy cost of $\sim 90$ kilocalories, or 1.24 kilocalories $\cdot \mathrm{kg}^{-1} \cdot$ day $^{-1}$ (Caruso and Hernandez, 2002). Two seated 3-set 8-repetition leg press workouts, whereby subjects $(\mathrm{n}=34)$ exerted either concentric-only or concentric and eccentric forces, showed muscle lengthening did not raise net energy costs (concentric-only $87.2 \pm 4.6$ kilocalories, concentric and eccentric $86.1 \pm 4.8$ kilocalories) despite eliciting an extra $\sim 3600$ joules of work (Caruso et al., 2003). Muscle lengthening likely entailed greater knee extensor series elastic element activity to yield such outcomes (Caruso et al., 2003). This is an important issue, as the ability to exert sufficeint eccentric forces were deemed crucial to emergency egress and the perfomance of in-flight operational tasks (Dudley et al., 1991: Stauber, 1989). Flywheel-based hardware also addresses many of the hardware concerns for exercise done within a spacecraft environment (Fitts et al., 2000; Matsumoto et al., 2011; Nicogossian et al., 1994). Yet prior flywheelbased research has focused on musculoskeletal/metabolic outcomes; future trials may wish to examine its utility to limit other types of physiological losses seen with spaceflight.

Due to their promise in ground-based research, flywheel-based devices are used on manned spaceflights. In addition there are several flywheel-based resistive exercise devices now in development at NASA's Glenn and Johnson Space Ceners, with the intent of their use as inflight hardware (International Countermeasures Working Group, 2010). Now aboard the ISS are two resistive exercise devices equipped with flywheels. A flywheel exercise device (FWED), 


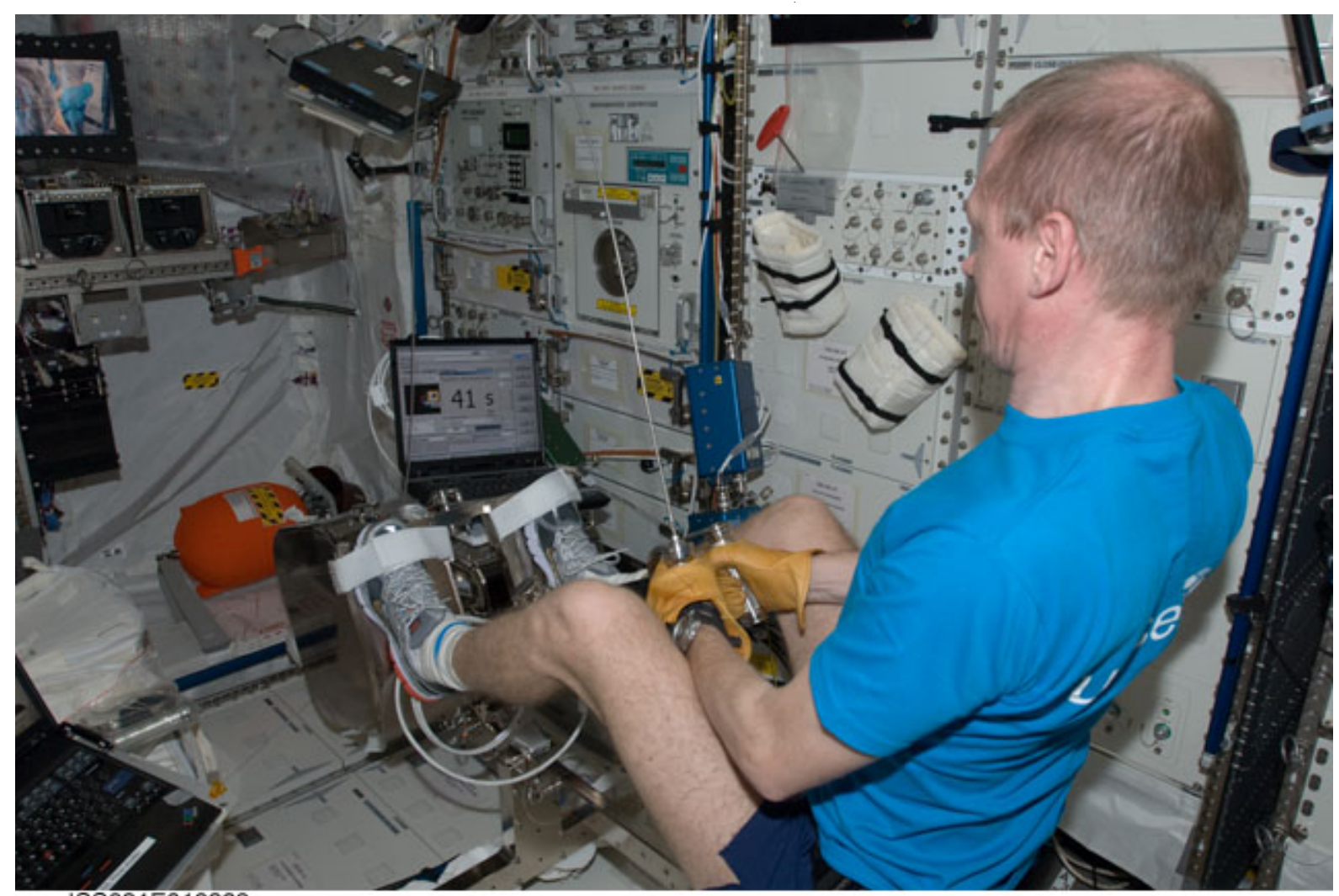

ISS021E016863

Figure 1. The Flywheel Exercise Device (FWED) housed within the ISS's European Columbus Module.

put there by The European Space Agency, is housed within the ISS's European Columbus Module (International Countermeasures Working Group, 2010). It is the in-flight version of a prototype from which much ground-based data was obtained (Alkner and Tesch, 2004a; Alkner and Tesch, 2004b; Caruso et al. 2005b; Tesch et al., 2004). Figure 1 shows the FWED in use on the ISS.

The second piece of flywheel-based hardware is NASA's Advanced Resistive Exercise Device. Pneumatic cylinders are its primary source of resistance, while its flywheels simulate the inertial characteristics of lifting weights on Earth (Bentley et al., 2006). Due to the high degree of inertial resistance flywheel-based hardware imparts during repetitions, much of the prior research with this exercise modality examined musculoskeletal changes. Thus in terms of physiological losses, the remainder of this paper will focus on musculoskeletal changes produced by flywheel-based hardware in spaceflight/unloading models

\section{Mitigation of muscle atrophy and strength losses from flywheel-based hardware}

The prototype of the European Space Agency's in-flight device uses two flywheels to impart resistance during seated leg and calf press repetitions. Bed rest studies, 29-90 days in length, assessed the merits of the prototype flywheel ergometer to abate knee extensor mass and strength losses (Alkner and Tesch, 2004a; Alkner and Tesch, 2004b; Tesch et al., 2004). Results showed concurrent workouts on the prototype ergometer preserved knee extensor mass and elicited strength gains up to $7.7 \%$ higher than preunloading values, while bed rest control subjects who did not exercise had significant mass (-9$18 \%$ ) and strength (-30-45\%) deficits (Alkner and Tesch, 2004a; Alkner and Tesch, 2004b; Tesch et al., 2004). Yet other studies that used to the same prototype flywheel ergometer produced different results (Caruso et al. 2004b; Caruso et al. 2005b). Three groups of subjects underwent 40 days of unilateral limb suspension (Caruso et al., 2004b; 
Caruso et al., 2005b). Two groups exercised on the ergometer three days per week, while a third served as unloaded controls. The two flywheel ergometer groups also received a capsule dosing assignment; subjects either consumed a placebo (lactose) or $16 \mathrm{mg} \cdot \mathrm{day}^{-1}$ of albuterol with no crossover. Results showed the combined flywheel ergometer-placebo treatment group preserved knee extensor mass, yet over time that group incurred significant (-10\%) concentric strength losses over the 40-day unloading period that were less than those for the unloaded controls (Caruso et al., 2004b). Yet subjects who received the combined flywheel ergometer-albuterol treatment acquired significant increases in concentric $(+18 \%)$ and eccentric $(+10 \%)$ total work over preunloading values (Caruso et al., 2004b). Ankle extensor data from the same three groups showed the combined flywheel ergometer-placebo treatment led to significant intra-group ( -13$15 \%)$ eccentric strength losses over 40 days (Caruso et al., 2005b). Yet subjects who received the flywheel ergometer-albuterol treatment had significant eccentric strength (+23-26\%) increases over the 40-day unloading period (Caruso et al., 2005b). More than one reason may account for differences in the flywheel-only outcomes for the 40-day trial and bed rest studies. Motivation levels, which in part resulted from differences in the study designs, may account for much of this discrepancy. For the unilateral limb suspension study, subjects were double-blinded to the capsule assignment and may have led some to erroneously believe they could rely on albuterol's ergogenic effect to abate strength losses. Other studies (Alkner and Tesch, 2004a; Alkner and Tesch, 2004b; Tesch et al., 2004), where only one treatment was administered, subjects knew they must rely solely upon the ergometer to maintain muscle mass and strength, and thus may have been more motivated for workouts.

\section{Mitigation of bone losses from flywheel-based hardware}

Prior research questioned if in-flight hardware offered a mechanical loading stimulus of sufficient intensity to abate bone losses (Cavanaugh et al., 2005; Schneider et al., 2003; Shackelford et al., 2005). Due to its ability to abate muscle mass and strength losses in groundbased trials, research assessed the merits of the prototype flywheel ergometer to mitigate bone deficits. A 90-day bed rest study examined subjects concurrently assigned to: 1) prototype flywheel ergometer workouts done every 2-3 days, 2): pamidronate therapy to block bone resorption, or 3): a control (no exercise or drug) condition with no crossover (Rittweger et al., 2005). Results showed ergometer workouts concurrent to bed rest abated calf cross-sectional area losses, while tibial diaphyseal and epiphyseal bone mineral content deficits were abated when pamidronate was used as a countermeasure. Each treatment was only partially mitigated bed restinduced lower leg losses, and the mechanical loading from the prototype ergometer was thought essential to bone health (Rittweger et al., 2005). Due to the merits of combined prototype ergometer-albuterol administrations on muscle strength, this same treatment was also assessed for its ability to abate unloading-induced bone losses (Caruso et al., 2004a). Subjects performed 40 days of unilateral limb suspension with their left legs, which otherwise refrained from traditional weight-bearing activity. As subjects performed concurrent workouts on the prototype flywheel ergometer three days ${ }^{\text {week }}{ }^{-1}$ with their left legs, they either consumed placebo capsules or a $16 \mathrm{mg}$ - day $^{-1}$ dose of albuterol with no crossover. Before and after the 40-day period bone densitometry quantified left leg changes. Mechanical loading values from the ergometer workouts were also analyzed. A significant time effect (pre > post) occurred for pelvic bone mineral density ($1.95 \%$ ); this was the first study to see significant skeletal losses from unilateral limb suspension (Caruso et al., 2004a). Those in the combined flywheel ergometer-albuterol group incurred significant bone mineral content gains to their unloaded leg and a trend (+2.9\%) for higher muscle mass, while the flywheel ergometerplacebo group produced no change to those variables. For the latter stages of the 40-day period, mechanical loads for prototype ergometerplacebo subjects declined significantly $(-14 \%)$ as compared to intra-group values from the start of unloading period. It was suggested albuterol augmented ergometer workouts to maintain mechanical loads that led to bone mineral content gains to the unloaded leg (Caruso et al., 2004a).

Some unloaded leg variables were maintained throughout the 40-day period in the 
flywheel ergometer-placebo group (Caruso et al., 2004a). Yet a comparative study showed the ergometer as a sole treatment does not promote bone accretion (Caruso et al., 2005a). With a matched-pairs design, ambulatory subjects were assigned to ten weeks of leg press workouts on the prototype ergometer or a standard leg press device with no crossover (Caruso et al., 2005a). Each group performed identical workout protocols and refrained from all other exercise. The progressive overload incurred by the groups was similar over ten weeks. Significant time (pre < post) effects for concentric knee extensor strength, leg muscle mass, body fat percentage and total fat mass occurred for both groups. Yet bone mineral density data produced group-by-time interactions, as standard resistive exercise evoked significant $(+1.2 \%)$ gains for both leg and total-body bone mineral density while the prototype ergometer group incurred no change. Bone resorption assays showed insignificant changes. It was concluded, that unlike standard resistive exercise, the mechanical stimuli provided by the ergometer does not impart strains upon bone of a sufficient magnitude and rate to evoke osteogenesis (Caruso et al., 2005a). Study results, whereby it was concluded strain rates impeded bone improvements from workouts done on flywheelbased hardware, illustrate a potential paradox for this exercise modality. While larger flywheels impart greater inertial resistance for a more intense mechanical loading stimulus to muscles, it occurs at the expense of reduced strain rates to bones. Even early papers that extolled the potential of flywheel-based hardware to abate muscle atrophy and strength losses, yet were more reserved in regards to its ability to abate in-flight bone deficits (Berg and Tesch, 1994). Subsequent research results, presented in this paper, appear to support this concern. Less resistance, provided by smaller flywheels, may permit the type of bone strains seen with impulse loading. Best achieved with repetitions done at higher speeds, and defined as the force per unit time, impulse loading may elicit bone mineral density increases over time (Dériaz et al., 2010; Heikkinen et al., 2007).

Unlike the European Space Agency's flywheel-based hardware, NASA produced no prototype of the Advanced Resistive Exercise Device from which bone data were obtained. Yet limited in-flight data was obtained on that device's impact on bone mineral changes during extended (4-6 month) stays aboard The ISS (Smith et al., 2012a). Crewmembers exercised with either the Advanced $(n=5)$ or Interim ( $=$ 8) Resistive Exercise Devices, the latter's hardware did not have flywheels and imparted comparatively lighter peak loads (Smith et al., 2012a). Data, obtained before and 5-45 days after spaceflights, showed bone mineral content and densities were best preserved in crewmembers who used the Advanced Resistive Exercise Device. For some bone-based dependent variables the inter-group differences were significant despite the modest sample sizes (Smith et al., 2012a). It was reported all crewmembers had nominal vitamin D status before and during spaceflight. It was concluded that with improved nutrition and exercise hardware, spaceflightinduced bone losses could be mitigated (Smith et al., 2012a).

\section{CONCLUSIONS}

Evidence from spaceflight/unloading models shows flywheel-based resistive exercise hardware is efficacious as a countermeasure to muscle mass and strength losses. Exercise done on flywheelbased hardware produced minimal increases in net energy costs (Caruso et al., 2003; Caruso and Hernandez, 2002). Yet it has generally not been as successful in addressing concenrs related to bone. Since flywheel-based hardware imparts a large amount of resistance, repetitions proceed at slow rates that in turn impede the rate of strain imparted to the bone segments engaged in exercise. Thus more success as a bone loss countermeasure may be achieved with exercise hardware that imparts the type of bone strains seen with impulse loading. Due to its ability to reduce muscle atrophy and strength losses with little increase in net energy costs, in-flight flywheel-based hardware should perhaps be redesigned so it imparts greater strain rates to bone for exercise done in $\mu g$. Such new hardware should also be examined as a countermeasure to other physiological losses seen with $\mu g$.

\section{REFERENCES}

Alkner BA, Tesch PA (2004a) Efficacy of a gravity-independent resistance exercise device as a countermeasure to muscle 
atrophy during 29-day bed rest. Acta Physiologica Scandinavica 181: 345-357

Alkner BA, Tesch PA (2004b) Knee extensor and plantar flexor muscle size and function following 90 days of bed rest with or without resistance exercise. European Journal of Applied Physiology 93: 294-305

Baldwin KM, White TP, Arnaud SB, Edgerton VR, Kraemer WJ, Kram R, Raab-Cullen D, Snow CM (1996) Musculoskeletal adaptations to weightlessness and development of effective countermeasures. Medicine and Science in Sports and Exercise 28: 1247-1253

Bamman MM, Hunter GR, Stevens BR, Guilliams ME, Greenisen MC (1998) Imapct of resistance exercise during bed rest on skeletal muscle sarcopenia and myosin isoform distribution. Journal of Applied Physiology 84: 157-163

Belavy DL, Miolovic T, Armbrecht G, Richardson CA, Rittweger J, Felsenberg D (2009) Differential atrophy of the lower limb musculature during prolonged bed rest. European Journal of Applied Physiology 107: 489-499

Bentley JR, Leach MA, McCleary F, Smith C, Norcross J, Hagan RD, Dao A, Zamaitis J, Landeck M, Groat M, Carrejo D, Cooper J, Weisskopf G, Mangieri M (2006) Advanced Resistive Exercise Device (ARED) Man-InThe-Loop Test (MILT). NASA/TP-2206213717

Berg HE, Dudley GA, Haggmark T, Ohlsen H, Tesch PA (1991) Effects of lower limb unloading on skeletal muscle mass and function in humans. Journal of Applied Physiology 70: 1882-1885

Berg HE, Eiken O, Miklavcic L, Mekjavic IB (2007) Hip, thigh, and calf muscle atrophy and bone loss after 5-week bed rest inactivity. European Journal of Applied Physiology 99: 283-289

Berg HE, Tesch PA (1994) A gravity-independent ergometer to be used for resistance training in space. Aviation Space and Environmental Medicine 65:752-756

Blanc S, Normand S, Ritz P, Pachiaudi C, Vico L, Gharib C, Gauquelin-Koch G (1998) Energy and water metabolism, body composition, and hormonal changes induced by 42 days of enforced inactivity and simulated weightlessness. Journal of Clinical Endocrinology and Metabolism 83: 4289-4297

Carpenter RD, LeBlanc AD, Evans H, Sibonga JD, Lang TF (2010) Long-term changes in the density and structure of the human hip and spine after long-duration spaceflight. Acta Astronautica 67: 71-81

Caruso JF, Coday MA, Monda JK, Ramey ES, Hastings LP, Vingren JL, Potter WT, Kraemer WJ, Wickel EE (2010) Blood lactate and hormonal responses to prototype flywheel ergometer workouts. Journal of Strength and Conditioning Research 24: 749-756

Caruso JF, Hamill JL, Hernandez DA, Yamauchi M (2005a) A comparison of isoload and isoinertial leg press training on bone and muscle outcomes. Journal of Strength and Conditioning Research 19: 592-598

Caruso JF, Hamill JL, Yamauchi M, Cook TD, Mercado DR, Gibb G, Higginson B, Elias J, Hernandez DA (2004a) Can albuterol help resistance exercise attenuate unloadinginduced bone losses? Journal of Strength and Conditioning Research 18:753-759

Caruso JF, Hamill JL, Yamauchi M, Mercado DR, Cook TD, Keller CP, Montgomery AG, Elias J (2004b) Albuterol helps resistance exercise attenuate knee extensor unloadinginduced strength losses. Aviation, Space and Environmental Medicine 75: 505-511

Caruso JF, Hamill JL, Yamauchi M, Mercado DR, Cook TD, Higginson B, O'Meara S, Elias J, Siconolfi SF (2005b) Albuterol augments resistance exercise to attenuate ankle extensor strength loss. Journal of Applied Physiology 98:1705-1711

Caruso JF, Hernandez DA, Saito K, Cho M, Nelson NM (2003) Inclusion of eccentric actions on metabolic cost resulting from work done on an inertial resistance ergometer. Journal of Strength and Conditioning Research 17: 549-555

Caruso JF, Hernandez DA (2002) The metabolic cost of a multi-set leg press protocol performed on a flywheel ergometer. Journal of Strength and Conditioning Research 16: 567-572 
Cavanaugh PR, Licata AA, Rice AJ (2005) Exercise and pharmacological countermeasures for bone loss during longduration space flight. Gravitational and Space Biology 18: 39-58

Collet P, Uebelhart D, Vico L, Moro L, Hartmann D, Roth M, Alexandre C (1997) Effects of 1- and 6-month spaceflight on bone mass and biochemistry in two humans. Bone 20: 547-551

Convertino VA (1990) Physiological adaptations to weightlessness: effects on exercise and work performance. Exercise and Sport Science Reviews 18: 119-166

Convertino VA, Doerr DF, Mathes KL, Stein SL, Buchanan P (1989) Changes in volume, muscle compartment, and compliance of the lower extremities in man following 30 days of exposure to simulated microgravity. Aviation Space and Environmental Medicine 60: 653-658

Crandall CG, Shibaski M, Wilson TE, Levine BD (2003) Prolonged head-down tilt exposure reduces maximal cutaneous vasodilator and sweating capacity in humans. Journal of Applied Physiology 94: 2330-2336

Cree MG, Paddon-Jones D, Newcomer BR, Ronsen O, Aarsland A, Wolfe RR, Ferrando AA (2010) Twenty-eight-day bed rest with hypercortisolemia induces peripheral insulin resistance and increases intramuscular triglycerides. Metabolism: Clinical and Experimental 59: 703-710

Crucian BE, Cubbage ML, Sams CF (2000) Altered cytokine production by specific human peripheral blood cell subsets immediately following space flight. Journal of Interferon and Cytokine Research 20: $547-556$

Delp MD (2007) Arterial adaptations in microgravity contribute to orthostatic tolerance. Journal of Applied Physiology 102: 853-858

Dériaz O, Najafi B, Ballabeni P, Crettenand A, Gobelet C, Aminian K, Rizzoli R, Gremion (2010) Proximal tibia volumetric bone mineral density is correlated to the magnitude of local acceleration in male long-distance runners. Journal of Applied Physiology 108: 852-857
Dudley GA, Tesch PA, Miller BJ, Buchanan P (1991) Importance of eccentric actions in performance adaptations to resistance training. Aviation Space and Environmental Medicine 62: 543-550

Edgerton VR, Zhou M-Y, Ohira Y, Klitgaard H, Jiang B, Bell G, Harris B, Saltin B, Gollnick PD, Roy RR, Day MK, Greenisen M (1995) Human fiber size and enzymatic properties after 5 and 11 days of space flight. Journal of Applied Physiology 78: 1733-1739

Ferrando AA, Stuart CA, Brunder DG, Hillman GR (1995) Magnetic resonance imaging quantitation of changes in muscle volume during seven days of strict bed rest. Aviation Space and Environmental Medicine 66: 976-981

Ferretti G, Capelli C (2009) Maximal $\mathrm{O}_{2}$ consumption: Effects of gravity withdrawal and resumption. Respiratory Physiology and Neurobiology, 169: S50-S54

Fitts RH, Riley DR, Widrick JJ (2000) Physiology of a microgravity environment invited review: microgravity and skeletal muscle. Journal of Applied Physiology 89: 823-839

Fortney SM, Mikhaylov V, Lee SM, Kobzev Y, Gonzalez RR, Greenleaf JE (1998) Body temperature and thermoregulation during submaximal exercise after 115-day spaceflight. Aviation Space and Environmental Medicine 69: 137-141

Friman G, Hamrin E (1976) Changes of reactive hyperaemia after clinical bed rest for seven days. Upsala Journal of Medical Sciences 81: 79-83

Gopalakrishnan R, Genc KO, Rice AJ, Lee SM, Evans HJ, Maender CC, Ilaslan H, Cavanagh PR (2010) Muscle volume, strength, endurance, and exercise loads during 6-month missions in space. Aviation, Space and Environmental Medicine 81: 91102

Hargens AR, Richardson S (2009) Cardiovascular adaptations, fluid shifts, and countermeasures related to space flight. Respiratory Physiology and Neurobiology, 169: S30-S33

Hawkey A (2005) Physiological and biomechanical considerations for a human Mars mission. Journal of the British Interplanetary Society 58: 117-130 
Hayes JC, Roper ML, Mazzocca AD, McBrine JJ, Barrows LH, Harris BA, Siconolfi SF (1992) Eccentric and concentric muscle performance following seven days of simulated weightlessness. NASA Tech Paper 3182

Heikkinen R, Vihriälä E, Vainionpää A, Korpelainen R, and Jämsä T (2007) Acceleration slope of exercise-induced impacts is a determinant of changes in bone density. Journal of Biomechanics 40: 29672974

Hikida RS, Gollnick PD, Dudley GA, Convertino VA, Buchanan P (1989) Structural and metabolic characteristics of human skeletal muscle following 30 days of simulated microgravity. Aviatiation Space and Environmental Medicine 60: 664-670

Holick MF (1998) Perspective on the impact of weightlessness on calcium and bone metabolism. Bone 22: 105S-111S

International Countermeasures Working Group, Trieste, Italy (2010) http://www.nasa.gov/ centers/johnson/pdf/505724main_Internatio nal_Exercise_Countermeasures_Hardware_ Catalog.pdf

Iwate S (2005) Effectiveness of centrifugeinduced artificial gravity with ergometric exercise as a countermeasure during simulated microgravity exposure in humans. Acta Astronautica 57: 75-80

Lang T, LeBlanc AD, Evans H, Lu Y, Genant H, Yu A (2004) Cortical and trabecular bone mineral loss from the spine and hip in longduration space flight. Journal of Bone and Mineral Research 19: 1006-1012

LeBlanc AD, Schenider VS, Evans H, Pientok C, Rowe R, Spector E (1992) Regional changes in muscle mass following 17 weeks of bed rest. Journal of Applied Physiology 73: $2172-2178$

Matsumoto A, Storch KJ, Stolfi A, Mohler SR, Frey MA, Stein TP (2011) Weight loss in humans in space. Aviation, Space, and Environmental Medicine 82: 615-621

Matsuo T, Ohkawara K, Seino S, Shimojo N, Yamada S, Ohshima H, Tanaka K, Mukai C (2012) An exercise protocol designed to control energy expenditure for long-term space missions. Aviation Space and Environmental Medicine 83: 783-789
Moore AD, Lee SMC, Stenger MB, Platts SH (2010) Cardiovascular exercise in the U.S. space program: Past, present and future. Acta Astronautica 66: 974-988

Morgan JLL, Zwart SR, Heer MA, Ploutz-Snyder L, Ericson K, Smith SM (2012) Bone metabolism and nutritional status during 30day head-down-tilt bed rest. Journal of Applied Physiology 113: 1519-1529

Nicogossian AE, Huntoon CL, Pool SL (1994) Space physiology and medicine, Philadelphia: Lea and Feiberger

Oganov VS, Cann C, Rakmanov AS, Ternovoy SK (1990) Computer tomography measurements of vertebral bones and muscles following extended manned space flight missions. Kosmicheskaya Biologiya $i$ Aviakosmicheskaya Meditsina 24: 20-21

Ogawa Y, Iwasaki K, Aoki K, Saitoh T, Kato J, Ogawa S (2009) Dynamic cerebral autoregulation after mild dehydration to simulate microgravity effects. Aviation Space and Environmental Medicine 80: 443-447

Parmar PJ, Perry RA, Martin JL, Vickers SD, Baptista RA, Edwards R, McArtor JD, Mitchell LN, Caruso JF (2015). Net energy costs from resistive exercise done on flywheel-based hardware. Isokinetics and Exercise Science 23: 221-227

Riley DA (1999) Is skeletal muscle ready for long-term spaceflight and return to gravity? Advances in Space Biology and Medicine 7: 31-48

Riley DA, Bain JL, Thompson JL, Fitts RH, Widrick JJ, Trappe SW, Trappe TA, Costill DA (2002) Thin filament diversity and physiological properties of fast and slow fiber types in astronaut leg muscles. Journal of Applied Physiology 92: 817-825

Rittweger J, Frost HM, Schiessl H, Ohshima H, Alkner BA, Tesch PA, Feisenberg D (2005) Muscle atrophy and bone loss after 90 days' bed rest and the effect of flywheel resistive exercise and pamidronate: results from the LTBR study. Bone 36: 1019-1029

Rittweger J, Winwood K, Seynnes O, de Boer M, Wilks D, Lea R, Rennie M, Narici M (2006) Bone loss from the human distal tibia epiphysis during 24 days on unilateral lower 
limb suspension. Journal of Physiology 577: 331-337

Ronca AE, Baker ES, Bavendam TG, Beck KD, Miller VM, Tash JS, Jenkinds M (2014) Effects of sex and gender on adaptations to space: reproductive health. Journal of Women's Health 23: 967-974

Schneider SM, Amonette WE, Blazine K, Bentley J, Lee SMC, Loehr JA, Moore AD, Rapley M, Mulder ER, Smith SM (2003) Training with the International Space Station interim resistive exercise device. Medicine and Science in Sports and Exercise 35: 19351945

Schulze K, Gallagher P, Trappe S (2002) Resistance training preserves skeletal muscle function during unloading in humans. Medicine and Science in Sports and Exercise 34: 303-313

Shackelford LC, LeBlanc AD, Driscoll TB, Evans HJ, Rianon NJ, Smith SM, Spector E, Feeback DL, Lai D (2005) Resistance exercise as a countermeasure to disuseinduced bone loss. Journal of Applied Physiology 97: 119-129

Shenkman BS, Nemirovskaya TL (2008) Calcium-dependent signaling mechanisms and soleus fiber remodeling under gravitational unloading. Journal of Muscle Research and Cell Motility 29: 221-230

Sibonga JD, Cavanagh PR, Lang TF, LeBlanc AD, Schneider VS, Shackelford LC, Smith SM, Vico L (2007) Adaptation of the skeletal system during long-duration space flight. Clinical Reviews of Mineral Metabolism 5: 249-261

Smith SM, Davis-Street JE, Rice BL, Nillen JL, Gillman PL, Block G (2001) Nutritional status assessment in semiclosed environments: Ground-based and space flight studies in humans. Journal of Nutrition 131: 2053-2061

Smith SM, Heer MA, Shackelford LC, Sibonga JD, Ploutz-Snyder L, Zwart SR (2012a) Benefits for bone from resistive exercise and nutrition in long-duration spaceflight: evidence from biochemistry and densitometry. Journal of Bone and Mineral Research 27: 1896-1906

Smith SM, Heer MA, Wang Z, Huntoon CL, Zwart SR (2012b) Long-duration space flight and bed rest effects on testosterone and other steroids. The Journal of Clinical Endocrinology and Metabolism, 97: 270278

Smith SM, McCoy T, Gazda D, Morgan JLL, Heer M, Zwart SR (2012c) Space flight calcium: implications for astronaut health, spacecraft operations, and Earth. Nutrients, 4: 2047-2068

Smith SM, Rice BL, Dlouhy H, Zwart SR (2013) Assessment of Nutritional Intake During Space Flight and Space Flight Analogs. Procedia Food Science 2: 27-34

Smith SM, Zwart SR (2008) Chapter 3: Nutritional Biochemistry of Spaceflight. In Gregory S. Makowski (Ed.), Advances in Clinical Chemistry 46: 87-130

Smith SM, Zwart SR, Block G, Rice BL, DavisStreet JE (2005) Nutritional status assessment of International Space Station crewmembers. Journal of Nutrtion 135: 437-443

Stauber WT (1989) A unique problem of muscle adaptation from Weightlessness: The deceleration deficiency. NASA Johnson Space Center, Workshop on Exercise Prescrition for Long-Duration Space Flight; p 57-59, (SEE N91-10574 01-52)

Stein TP (2000) The relationship between dietary intake, exercise, energy balance and the space craft environment. Pflugers Archives 441: R21-R31

Stein TP, Leskiw MJ (2000) Oxidant damage during and after spaceflight. American Journal of Physiology Endocrinology and Metabolism 278: E375-E382

Stein TP (2002) Space flight and oxidative stress. Nutrition 18: 867-871

Stein TP, Leskiw MJ, Schulter MD, Hoyt RW, Lane HW, Gretebeck RE, Le Blanc AD (1999) Energy expenditure and balance during spaceflight on the space shuttle. American Journal of Physiology 276: R1739-R1748

Stowe RP, Mehta SK, Ferrando AA, Feeback DL, Pierson DL (2001) Immune responses and latent herpesvirus reactivation in spaceflight. Aviation Space and Environmental Medicine 72: 884-891

Stowe RP, Sams CF, Pierson DL (2003) Effects of mission duration on neuroimmunbe 
responses in astronauts. Aviation Space and Environmental Medicine 74: 1281-1284

Streeper T, Cavanagh PR, Hanson AM, Carpenter RD, Saeed I, Kornak J, Lang T (2011) Development of an integrated countermeasure device for use in longduration spaceflight. Acta Astronautica, 68: 2029-2037

Strollo F (1999) Hormonal changes in humans during spaceflight. Advances in Space Biology and Medicine. 7: 99-129

Tesch PA, Trieschmann JT, Ekberg A (2004) Hypertrophy of chronically unloaded muscle subjected to resistance exercise. Journal of Applied Physiology 96: 14511458

Trappe S, Costill D, Gallagher P, Creer A, Peters JR, Evans H, Riley DA, Fitts RH (2009) Exercise in Space: human skeletal muscle after 6 months aboard the International Space Station, Journal of Applied Physiology 106: 1159-1168
Whittle MW (1979) Caloric and exercise requirements of space flight: Biostereometric results from Skylab. Aviation Space and Environmental Medicine 50: 163-167

Wickman L, Nota B, Keates S (2011) Lunar life support system study: metabolic energy and water considerations. July $16^{\text {th }}, 2011$ from http://www.apu.edu/cris/pdfs/cris_lunar_life .$p d f$

Yang CB, Wang YC, Gao Y, Geng J, Wu YH, Zhang Y, Sun XQ (2011) Artificial gravity with ergometric exercise preserves the cardiac, but not cerebrovascular, functions during 4 days of head-down bed rest. Cytokine 56:, 648-655

Zwart SR, Gibson CR, Mader TH, Ericson K, Ploutz-Snyder L, Heer MA, Smith SM (2012) Vision changes after spaceflight are related to alterations in folate- and vitamin B-12-dependent one-carbon metabolism. The Journal of Nutrition 142: 427-431 